\title{
Research on Embedded System Design Based on ARM
}

\author{
Gaohong Yang \\ Xi' an International University, School of Applied Technology, Information experiment center, \\ ShanXi Xi'An, China
}

Keywords: Embedded System, Design, ARM

\begin{abstract}
In recent years, more and more rapid development of science and technology, computer network technology and the development of microelectronics technology, making embedded systems in a wide range of applications in various industries, the traditional embedded system is gradually exposed, ARM embedded system has gradually become the best choice for many industry embedded solutions. In this paper, the general situation of ARM embedded system is studied and its design method is obtained.
\end{abstract}

\section{Introduction}

From the single-chip to the present, its type has undergone great changes, a variety of high-performance SOC design and application, making mobile communications, instrumentation, industrial equipment, etc. has a new function, as the core of embedded systems, ARM processing Is the most advanced embedded microprocessor. The development of information technology makes people have a new demand for the function of network interconnection of embedded systems, and pay more attention to the network performance of embedded systems. Embedded system is based on the application to computer technology-based, to meet the application system on the price, reliability, size, power consumption and other aspects of the strict requirements of the special computer system. From the twentieth century, eighty years ago, some organizations and companies on the international community began to commercial embedded systems and dedicated operating system research and development. But the initial development of the embedded system is only a simple function of the weak products, can only provides some of the initial management functions. With the development of information technology and semiconductor technology, the price of 32-bit microprocessors and 8-bit machines have been similar, and sometimes from the overall cost of the system to consider the 32-bit processor system costs will be lower, and 32-bit processing resource resources than the 8-bit machine is much more abundant, which will make 32-bit processor has become the mainstream of the market. Now with the embedded system to enhance the function of the program code becomes more and more complex, the traditional software design is difficult to ensure the reliability and stability of the system, the use of embedded operating system as a software development platform is to solve this problem way. There are dozens of developers at home and abroad embedded real-time operating system, they can provide real-time better, powerful kernel, and provide editing, compilation and simulation of the integrated development environment. At present, the embedded system is in a period of rapid development, technology and market, the application of embedded systems will become one of the hot spots of the twenty-first century. This paper mainly studies the design of embedded system based on ARM.

\section{General Overview of Embedded Systems}

Embedded system is mainly used to monitor, control or help the operation of machines and equipment, embedded system is a combination of hardware and software systems, embedded systems for the general definition of application-centric, computer technology-based to achieve the application system Performance, cost, size of the demand, the embedded system is divided into three stages: single-chip stage, embedded CPU and embedded operating system phase and SOC and 
network stage. For the single-chip stage is mainly a simple function of the microcontroller as the core, and did not use embedded operation, the system structure and function is more single. In the embedded CPU and embedded operation stage, a variety of embedded micro-processor and embedded operations appear, making the application of embedded systems more widely in the defense, industry and agriculture and transportation applications. In SOC and network stage, SOC-based embedded system is more powerful, cost and energy consumption is getting smaller and smaller, in people's daily life more and more widely.

\section{Research on the Development of ARM Processor}

Embedded micro-processor as the core of the entire embedded system, in the design, should be based on the needs of the application, after years of development, ARM has developed a series of ARM processor core, the current application of the most systems ARM7 series, ARM9 series, ARM11 series and Cortex series, the future development trend of ARM system mainly in: high-performance ARM core, these microprocessors are mainly used in professional fields, for high-performance ARM core, for complex application, the cost of investment for the ARM core is also reduced to meet the high-performance, low-cost basis, greatly improve the performance of ARM.

\section{Design Strategy of Embedded Hardware and Software System Based on ARM}

Hardware System Design. The choice of embedded microprocessors ARM chip has three main models, the choice of ARM core is the decisive factor is whether to bring their own MMU function, part of the ARM core only with the MMU function under the premise can run, so in the Special attention should be paid to this function when selecting, for priority systems that do not require MMU support. Second, the ARM system clock frequency should be observed, the system clock frequency is to determine the ARM chip processing speed of the direct factors for the higher frequency of the ARM chip, represented by ARM9, the frequency can reach $400 \mathrm{MHz}$, in the selection should be based on the operation of the entire system to decide. But also should be considered is the ARM chip built-in external interface controller number, for the ARM chip should choose to expand the RS232 serial interface, Ethernet interface, etc., the best integrated DMA controller to ensure a large number of high-speed data transmission.

The hardware of this paper is based on Samsung S3C44B0X microprocessor (ARM7) as the core of the motherboard, the interface is very rich, to meet the needs of embedded technology system hardware, especially in the embedded technology course teaching experimental system applications. The system uses Samsung S3C44B0X with up to $66 \mathrm{MHz}$ frequency as the processor chip, which integrates various system interfaces, thus minimizing system development time and cost and reducing the use of peripheral devices. The hardware platform includes the following three modules: (1) the minimum system: power circuit, crystal circuit, reset circuit and storage system; (2) man-machine dialogue: touch screen, liquid crystal display and audio module; (3) communication module: Serial interface and JTAG debug interface, USB module and Internet module

In general, the ARM system as the core of the hardware and software design should be a multi-platform support for embedded platforms, so the overall design of the hardware system, in order to facilitate a variety of ARMCPU switch, should be in Chip design of some small sub-board, for the design of peripheral circuits, the mother board should be through the golden finger interface to connect, this design method has the advantage of small footprint, can greatly reduce the production costs.

Memory design planning is mainly carried out on the main memory and auxiliary memory size and model of choice for the main memory selection mainly on the processing speed and price considerations, for the larger memory system, SDRAM can be given priority. For the auxiliary memory, mainly used to store large amounts of data program code, so the size of the auxiliary memory is very important, which, NANDFLASH memory, low cost, is the primary object of choice. 
Software System Design. For the mainstream ARM system, including: BootLoader, system software, graphical user interface and the upper application of four levels of software, in the basic structure of the structure, it should be a holistic consideration, from different levels of the system on the most Preferably in order to achieve the overall effect of the optimization. ARM system software development uses ADS1.2 to complete the Bootloader program design. Bootloader programming is divided into two parts, (1) with assembly language to achieve: and S3C44B0X hardware initialization related tasks and programming, including masking interrupt, memory system (NOR FLASH, RAM, PSRAM) initialization, stack initialization, (2) C language to achieve: complete support for serial communication protocol (that is, serial port initialization), support network communication protocol (network control initialization), the $\mu \mathrm{C}$ / OS-II kernel image file and root file system image file RAM to run the task. Embedded operating systems include: VxWorks, Linux and WinCE, in the selection, the three operating systems should be the advantages and disadvantages of in-depth study to understand its basic characteristics, in the selection can be targeted to design, software systems operating design, Linux is the fastest growing, most widely used, and its main performance is higher, as its preferred important factor. WinCE is from the whole for the limited resources for multi-threaded, full priority, multi-task operation, easy development, fast, but there are too shortcomings of the price. For VxWorks, it has a very good real-time feature and the price is very high, so the development of applications is not wide enough.

GUI system mainly includes: highly cut XWindows system, MiniGUI, OpenGUI, QT / embedded, etc., for each GUI has its own characteristics, in the selection is the need to carefully consider. For the first system, its use of the system in the process of more resources, coupled with the Chinese display and Chinese input and other local code use, making its consumption is more powerful, so mainly used in set-top boxes and other places. For the second system, it is mainly used to provide a lightweight graphical user interface support system, but it lacks a convenient integrated development environment. Therefore, in the ARM-based hardware and software system design should be combined with a variety of conditions for comprehensive consideration. 2 virtual experimental platform design In order to effectively enhance the depth of the content of the course, to improve the ability of students to use knowledge, to adapt to changes in the market, drawing on the results of previous generations, combined with the author's practical teaching experience, the use of simulation software Proteus and programming tools Keil, designed to complete a set of virtual experimental platform for open teaching. The virtual experiment platform uses the LPC2138 microcontroller based on ARM7 core as the core control chip, including 14 experimental modules, in addition to "assembly language experimental module", the other modules are used C language to achieve the preparation of software programs, basic experiment requirements based on the students can design their own procedures and modify.

Minimum System Experiment Module. The minimum system experimental module includes LPC2138 microcontroller, 3.3V power supply circuit, reset circuit and clock circuit, in which the clock circuit uses the oscillation mode to generate the clock signal. Learn to help students understand the concepts and components of the smallest system.

Assembly Language Experimental Module. Through a simple ARM assembler to achieve the two registers of the loop add. It allows students to understand the ARM assembly instructions format, program structure and basic style.

Input and Output (GPIO) Experimental Module. Using a pin to connect a button input and a buzzer and a LED output to complete the most basic GPIO input and output control. When the button press, the buzzer ring, LED light-emitting diode off; when the button is released, the buzzer does not ring, LED light-emitting diode light. In order to compare with the timing function in the timer experiment module, the delay function in this experiment module is realized by for loop statement.

External Interrupt Experiment Module. The output element uses a buzzer, two light-emitting diodes and the input element uses a button to simulate the external interrupt signal. Under normal conditions, two light-emitting diodes in turn, press the button to detect the interrupt signal, and then perform the interrupt processing function, after the completion of the implementation of the main function to continue to perform the light-emitting diode lighting task to simulate the interrupt flow. 
Timer Module. The main use of Timer 1 to achieve a comparison match the output control LED flashing, and the use of four-channel virtual oscilloscope A port to detect the output signal waveform. In order to familiarize students with the use of oscilloscopes and the comparison of various signals, the $\mathrm{B}, \mathrm{C}$, and $\mathrm{D}$ ports of the virtual oscilloscope enter the pulse signal, the clock signal and the sinusoidal signal.

SPI Experimental Module. Using a serial input and display driver chip 74HC595 control a LED digital tube cycle display $0 \sim$ F characters. Through the study can master the full-duplex synchronous serial interface SPI works, SPI interface circuit connection and LED digital display programming control method.

UART Experimental Module. Through the serial interface UART0 output string, the output string can be programmed to control, use the virtual terminal to receive and display, while using the virtual serial interface device COMPIM to the PC to send the string, and the PC terminal display received characters string to achieve micro-controller chip and PC communication. 2.9 ADC experimental module mainly through the adjustable resistor to change the input pin voltage to provide analog input, the experimental platform through the ADC on the analog external input voltage AD sampling conversion, and the results into voltage values, and then sent to the serial interface UART0 combined with the DV terminal in the experimental module to display.

\section{Conclusion}

Through the analysis of the functional requirements of embedded systems, targeted hardware and software system design to meet the needs of different industries for the microprocessor to achieve the purpose of solving practical problems, making the embedded system can be more widely used in communication Industry, to promote the development of computer network technology.

\section{References}

[1] Xiong Jiang. Embedded systems and pervasive computing[J]. SCM and embedded systems applications. 2003 (04)

[2] Xia Weiwei, Shen Lianfeng, Xiao Jie, Mao Yubin. Key technology analysis and application of embedded system [J] .Application of Single Chip Microcomputer and Embedded System 2003 (02)

[3] CUI Long, ZHOU Qi-ming, JIANG Wen-rui.Study on Real-time Measurement and Control System Development Platform Based on ARM [J] .Application of Single Chip Microcomputer and Embedded System 2003 (01)

[4] Gong Xiaohua. Embedded system design based on ARM [D]. Xi'an University of Technology 2007

[5] Wei Xing. ARM-based embedded system design method research [D]. Yanshan University 2007 